$$
k^{-n} P_{n}(k) \leqq 2^{n} \zeta^{-1}(n)+2^{n} \epsilon+o(1) .
$$

Since this is true for any $\epsilon$, one has

$$
k^{-n} P_{n}(k) \leqq 2^{n} \zeta^{-1}(n)+o(1),
$$

which gives together with (2) and (7) the relation (6).

\title{
REFERENCES
}

1. K. Mahler, On lattice points in n-dimensional star bodies, Proc. Roy. Soc. London vol. 87 (1946) pp. 151-187.

2. H. Minkowski, Geometrie der Zahlen, Leipzig, Teubner, 1910, p. 79.

3. C. A. Rogers, Existence theorems in the geometry of numbers, Ann. of Math. vol. 48 (1947) pp. 994-1002.

Oregon State College

\section{ON THE REPRESENTATION OF SEQUENCES AS FOURIER COEFFICIENTS}

\author{
P. G. ROONEY
}

If $F \in L_{p}(-\infty, \infty)$, where $1<p \leqq 2$, then $F$ has a Fourier transform $G \in L_{q}(-\infty, \infty)$, where $p$ and $q$ are connected, now and henceforth, by the relation

$$
p^{-1}+q^{-1}=1
$$

However, except in the case $p=2$, the collection of transforms does not cover $L_{q}(-\infty, \infty)$. In a recent paper [3], we gave a characterization of this collection of Fourier transforms.

A similar situation exists in the theory of Fourier series. If $F \in L_{p}(0,2 \pi)$, where $1<p \leqq 2$, and $c=\left\{c_{n}\right\}$ is the Fourier sequence of $F$, that is

$$
c_{n}=\frac{1}{2 \pi} \int_{0}^{2 \pi} F(t) e^{-i n t} d t
$$

then $[5, \S 9.1], c \in l_{q}$. That is

$$
\sum_{n=-\infty}^{\infty}\left|c_{n}\right|^{q}<\infty
$$

Received by the editors August 10, 1959 and, in revised form, December 11, 1959. 
However, the collection of Fourier sequences of functions in $L_{p}(0,2 \pi)$ does not cover $l_{q}-$ see $[1 ; 2]$. This collection has been characterized however in terms of the $(C, 1)$ summands of the member sequences - see $[5, \S 4.33]$.

Here we propose to give another characterization, which will be, in a sense, the discrete analogue of our theory for Fourier transforms. That theory depended on certain theorems concerning the representation of functions as Laplace transforms. Our theory here will depend on analogous theorems concerning the representation of sequences as Hausdorff moment sequences-the discrete anlogue of Laplace transforms.

In the theory of Hausdorff moment sequences, the functions

$$
\lambda_{k, m}(t)=C_{k, m} t^{m}(1-t)^{k-m}, \quad k=0,1,2, \cdots, 0 \leqq m \leqq k,
$$

play a crucial role-see $[4$, Chapter $3, \S 2$ et. seq. $]$. In our theory the numbers

$$
\begin{array}{r}
a_{r, k, m}=C_{k, m} \int_{0}^{1} t^{m}(1-t)^{k-m} e^{2 \pi i r t} d t, \quad r=0, \pm 1, \pm 2, \cdots, \\
k=0,1,2, \cdots, \quad 0 \leqq m \leqq k,
\end{array}
$$

which are essentially the Fourier coefficients of the functions $\lambda_{k, m}$, play a correspondingly important role. Our first efforts must be to derive certain properties of these numbers. We summarize these properties in the lemmas below, and then prove our representation theorem.

LEMMA 1.

$$
\begin{aligned}
a_{0, k, m} & =(k+1)^{-1} . \\
a_{r, k, k} & =\bar{a}_{r, k, 0} .
\end{aligned}
$$

If $r \neq 0$, then

$$
\begin{aligned}
a_{r, k, m}=\frac{k}{2 \pi i r}\left(a_{r, k-1, m}-a_{r, k-1, m-1}\right), & 0<m<k, \\
a_{r, k, k}=\frac{k}{2 \pi i r}\left(k^{-1}-a_{r, k-1, k-1}\right), & k>0, \\
a_{r, k, 0}=\frac{-k}{2 \pi i r}\left(k^{-1}-a_{r, k-1,0}\right), & k>0,
\end{aligned}
$$




$$
\begin{aligned}
& a_{r, k, k}=\left\{\begin{array}{lr}
(k+1)^{-1}, & r=0, \\
0, & k=0, r \neq 0, \\
\sum_{n=0}^{k-1} C_{k-1, n}(-1)^{n} \frac{n !}{(2 \pi i r)^{n+1}}, & k r \neq 0 .
\end{array}\right. \\
& a_{r, k, m}=C_{k, m}(-1)^{k-m} \Delta^{k-m} a_{r, m, m}
\end{aligned}
$$

(where $\Delta$ is the advancing difference operator operating on the $m$ variable: that is

$$
\Delta^{k} a_{m}=\sum_{n=0}^{k}(-1)^{n} C_{k, n} a_{m+k-n} .
$$

Proof. (4) and (5) are clear. (6), (7), and (8) follow from (3) by integration by parts. The first two parts of (9) are obvious and the last part follows from the second on repeated use of (7). For (10) we have

$$
\begin{aligned}
a_{r, k, m} & =C_{k, m} \int_{0}^{1} t^{m}(1-t)^{k-m} e^{2 \pi i r t} d t \\
& =C_{k, m} \sum_{n=0}^{k-m} C_{k-m, n}(-1)^{k-m-n} \int_{0}^{1} t^{k-n} e^{2 \pi i r t} d t \\
& =C_{k, m}(-1)^{k-m} \sum_{n=0}^{k-m}(-1)^{n} C_{k-m, n} a_{r, k-n, k-n} \\
& =C_{k, m}(-1)^{k-m} \Delta^{k-m} a_{r, m, m} .
\end{aligned}
$$

LEMMA 2.

$$
\left|a_{r, k, m}\right| \leqq(k+1)^{-1} .
$$

If $r \neq 0$,

$$
\left|a_{r, k, m}\right| \leqq(\pi|r|)^{-1} .
$$

Proof. (12) is obvious. For (13), if $r \neq 0,0<m<k$, we have from (6) and (12),

$$
\begin{aligned}
\left|a_{r, k, m}\right| & \leqq \frac{k}{2 \pi|r|}\left(\left|a_{r, k-1, m}\right|+\left|a_{r, k-1, m-1}\right|\right) \\
& \leqq \frac{k}{2 \pi|r|}\left(k^{-1}+k^{-1}\right)=(\pi|r|)^{-1} .
\end{aligned}
$$

Also if $r \neq 0, k>0$, we have from (5), (7) and (12), 


$$
\begin{aligned}
\left|a_{r, k, k}\right| & =\left|a_{r, k, 0}\right| \leqq \frac{k}{2 \pi|r|}\left(k^{-1}+\left|a_{r, k-1,0}\right|\right) \\
& \leqq \frac{k}{2 \pi|r|}\left(k^{-1}+k^{-1}\right)=(\pi|r|)^{-1} .
\end{aligned}
$$

Finally, if $r \neq 0$, then from (9) $\left|a_{r, 0,0}\right|=0 \leqq(\pi|r|)^{-1}$.

Lemma 3. If $c \in l_{q}, 1 \leqq q<\infty$, and $0 \leqq m \leqq k$, then $\sum_{-\infty}^{\infty} c_{r} a_{r, k, m}$ is convergent.

Proof. If $q>1$, then from Hölder's inequality for series, using (12) and (13),

$$
\begin{aligned}
\sum_{r=-\infty}^{\infty}\left|c_{r} a_{r, k, m}\right| & =\frac{\left|c_{0}\right|}{k+1}+\sum_{-\infty}^{\infty}\left|c_{r} a_{r, k, m}\right| \\
& \leqq \frac{\left|c_{0}\right|}{k+1}+\left\{\sum_{-\infty}^{\infty}\left|c_{r}\right|^{q}\right\}^{1 / q}\left\{2 \sum_{1}^{\infty} \frac{1}{r^{p}}\right\}^{1 / p}<\infty .
\end{aligned}
$$

If $q=1$, then

$$
\sum_{r=-\infty}^{\infty}\left|c_{r} a_{r, k, m}\right| \leqq \frac{1}{k+1} \sum_{-\infty}^{\infty}\left|c_{r}\right|<\infty .
$$

Let $c \in l_{q}, q \geqq 1,0 \leqq k \leqq m$. We can now define

$$
\mu_{k, m}(c) \equiv \sum_{-\infty}^{\infty} c_{r} a_{r, k, m}
$$

THEOREM. A necessary and sufficient condition that a sequence $c \in l_{q}$, $q \geqq 2$, be the Fourier sequence of a function in $L_{p}(0,2 \pi)$ is that there be a constant $M$ so that

$$
(k+1)^{p-1} \sum_{m=0}^{k}\left|\mu_{k, m}(c)\right|^{p} \leqq M, \quad k=0,1,2, \cdots .
$$

Proof of Necessity. Suppose $F \in L_{p}(0,2 \pi)$, where $1<p \leqq 2$, and

$$
c_{n}=\frac{1}{2 \pi} \int_{0}^{2 \pi} F(t) e^{-i n t} d t, \quad n=0, \pm 1, \pm 2, \cdots .
$$

Then

$$
F(t)=\underset{n \rightarrow \infty}{\mathfrak{L}_{p}} \sum_{-n}^{n} c_{r} e^{i r t}, \text { a.e., }
$$

where $\mathfrak{L}_{p}$ denotes the limit in mean of order $p$, and if $0 \leqq m \leqq k$, 


$$
\begin{aligned}
\mu_{k, m}(c) & =\sum_{r=-\infty}^{\infty} c_{r} a_{r, k, m}=\lim _{n \rightarrow \infty} \sum_{-n}^{n} c_{r} a_{r, k, m} \\
& =\lim _{n \rightarrow \infty} C_{k, m} \sum_{-n}^{n} c_{r} \int_{0}^{1} t^{m}(1-t)^{k-m} e^{2 \pi i r t} d t \\
& =\lim _{n \rightarrow \infty} C_{k, m} \int_{0}^{1} t^{m}(1-t)^{k-m}\left(\sum_{-n}^{n} c_{r} e^{2 \pi i r t}\right) d t \\
& =C_{k, m} \int_{0}^{1} t^{m}(1-t)^{k-m}\left(\underset{n \rightarrow \infty}{\mathcal{L}_{p}} \sum_{-n}^{n} c_{r} e^{2 \pi i r t}\right) d t \\
& =C_{k, m} \int_{0}^{1} t^{m}(1-t)^{k-m} F(2 \pi t) d t .
\end{aligned}
$$

Hence, using Hölder's inequality

$$
\begin{aligned}
\left|\mu_{k, m}\right| & \leqq C_{k, m} \int_{0}^{1} t^{m}(1-t)^{k-m}|F(2 \pi t)| d t \\
& \leqq C_{k, m}\left\{\int_{0}^{1} t^{m}(1-t)^{k-m}|F(2 \pi t)|^{p} d t\right\}^{1 / p}\left\{\int_{0}^{1} t^{m}(1-t)^{k-m} d t\right\}^{1 / q} \\
& =(k+1)^{-1 / q}\left\{C_{k, m} \int_{0}^{1} t^{m}(1-t)^{k-m}|F(2 \pi t)|^{p} d t\right\}^{1 / p} .
\end{aligned}
$$

Thus

$$
\begin{aligned}
\sum_{m=0}^{k}\left|\mu_{k, m}\right|^{p} & \leqq(k+1)^{-p / q} \sum_{m=0}^{k} C_{k, m} \int_{0}^{1} t^{m}(1-t)^{k-m}|F(2 \pi t)|^{p} d t \\
& =(k+1)^{-(p-1)} \int_{0}^{1}(t+(1-t))^{k}|F(2 \pi t)|^{p} d t \\
& =(k+1)^{-(p-1)} \int_{0}^{1}|F(2 \pi t)|^{p} d t,
\end{aligned}
$$

and hence there is such an $M$, namely

$$
M=\int_{0}^{1}|F(2 \pi t)|^{p} d t=\frac{1}{2 \pi} \int_{0}^{2 \pi}|F(t)|^{p} d t .
$$

Proof of sufficiency. Suppose $c \in l_{q}, q \geqq 2$; let

$$
\mu_{m}=\sum_{r=-\infty}^{\infty} c_{r} a_{r, m, m},
$$

and let

$$
\lambda_{k, m}=C_{k, m}(-1)^{k-m} \Delta^{k-m} \mu_{m} .
$$


Then from (10),

Thus

$$
\begin{aligned}
\lambda_{k, m} & =\sum_{r=-\infty}^{\infty} c_{r}\left\{C_{k, m}(-1)^{k-m} \Delta^{k-m} a_{r, m, m}\right\} \\
& =\sum_{r=-\infty}^{\infty} c_{r} a_{r, k, m}=\mu_{k, m}(c) .
\end{aligned}
$$

$$
(k+1)^{p-1} \sum_{m=0}^{k}\left|\lambda_{k, m}\right|^{p} \leqq M,
$$

and hence by $[4$, Chapter 3 , Theorem 5$]$, there is a function $g \in L_{p}(0,1)$ such that

$$
\mu_{m}=\int_{0}^{1} t^{m} g(t) d t, \quad m=0,1, \cdots .
$$

Let $F(t)=g(t / 2 \pi)$. Then $F \in L_{p}(0,2 \pi)$, and if $z$ is the Fourier sequence of $F$, then, operating formally, we obtain

$$
\begin{aligned}
\tilde{c}_{n} & =\frac{1}{2 \pi} \int_{0}^{2 \pi} F(t) e^{-i n t} d t=\int_{0}^{1} g(t) e^{-2 \pi i n t} d t \\
& =\sum_{m=0}^{\infty} \frac{(-2 \pi i n)^{m}}{m !} \int_{0}^{1} t^{m} g(t) d t=\sum_{m=0}^{\infty} \frac{(-2 \pi i n)^{m}}{m !} \mu_{m} \\
& =\sum_{m=0}^{\infty} \frac{(-2 \pi i n)^{m}}{m !} \sum_{r=-\infty}^{\infty} c_{r} a_{r, m, m} \\
& =\sum_{r=-\infty}^{\infty} c_{r} \sum_{m=0}^{\infty} \frac{(-2 \pi i n)^{m}}{m !} a_{r, m, m} \\
& =\sum_{r=-\infty}^{\infty} c_{r} \sum_{m=0}^{\infty} \frac{(-2 \pi i n)^{m}}{m !} \int_{0}^{1} t^{m} e^{2 \pi i r t} d t \\
& =\sum_{r=-\infty}^{\infty} c_{r} \int_{0}^{1} e^{2 \pi i(r-n) t} d t=c_{n} ;
\end{aligned}
$$

that is

$$
c_{n}=\frac{1}{2 \pi} \int_{0}^{2 \pi} F(t) e^{-i n t} d t,
$$

and $c$ is the Fourier sequence of a function in $L_{p}(0,2 \pi)$, provided that we justify the two interchanges of integration and summation, and the interchange of order of summation.

The interchanges of integration and summation are easily justified 
by the uniform convergence of the exponential series. For the interchange of order of summations, we must show that

$$
\Re_{n}=\sum_{r=-\infty}^{\infty}\left|c_{r}\right| \sum_{m=0}^{\infty}\left|\frac{(-2 \pi i n)^{m}}{m !} a_{r, m, m}\right|<\infty, \quad n=0, \pm 1, \pm 2, \cdots .
$$

But if $n \neq 0$, then from (12) and (13), and using Hölder's inequality,

$$
\begin{aligned}
\mathscr{K}_{n} & =\left|c_{0}\right| \sum_{m=0}^{\infty} \frac{(2 \pi|n|)^{m}}{m !}\left|a_{0, m, m}\right|+\sum_{-\infty}^{\infty}\left|c_{r}\right| \sum_{m=0}^{\infty} \frac{(2 \pi|n|)^{m}}{m !}\left|a_{r, m, m}\right| \\
& \leqq\left|c_{0}\right| \sum_{m=0}^{\infty} \frac{(2 \pi|n|)^{m}}{(m+1) !}+\frac{1}{\pi} \sum_{-\infty}^{\infty} \frac{\left|c_{r}\right|}{|r|} \sum_{m=0}^{\infty} \frac{(2 \pi|n|)^{m}}{m !} \\
& =\left|c_{0}\right| \frac{e^{2 \pi|n|}-1}{2 \pi|n|}+e^{2 \pi|n|}\left\{\sum_{-\infty}^{\infty}\left|c_{r}\right|{ }^{q}\right\}^{1 / q}\left\{2 \sum_{1}^{\infty} r^{-p}\right\}^{1 / p}<\infty,
\end{aligned}
$$

and

$$
\mathfrak{K}_{0}=\sum_{-\infty}^{\infty}\left|c_{r}\right|\left|a_{r, 0,0}\right|=\left|c_{0}\right|
$$

\section{REFERENCES}

1. G. H. Hardy and J. E. Littlewood, Some new properties of Fourier constants, Math. Ann. vol. 97 (1926) pp. 159-209.

2. Edwin Hewitt, Fourier transforms of the class $L_{p}$, Ark. Mat. vol. 2 (1954) pp. 571-574.

3. P. G. Rooney, On the representation of functions as Fourier transforms, Canad. J. Math. vol. 11 (1959) pp. 168-174.

4. D. V. Widder, The Laplace transform, Princeton, 1941.

5. A. Zygmund, Trigonometrical series, Warsaw, 1935.

UNIVERSITY OF TORONTO 